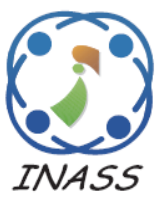

\title{
Electric Wheelchair Control Mechanism Using Eye-mark Key Point Detection
}

\author{
Gusti Pangestu $^{1 *} \quad$ Fitri Utaminingrum ${ }^{2}$ \\ ${ }^{1}$ Computer Vision Research Group, Faculty of Computer Science, Brawijaya University, Indonesia \\ * Corresponding author’s Email: Gustipangestu95@gmail.com
}

\begin{abstract}
People with deficiency hands and feet, especially people with multiple disabilities has difficulty for navigation. Therefore, this research proposed an approach to help them using an electric wheelchair and control it using their eyeball movements. However, eyeball movement detection is still a problem due to the lack of standardized methods such as low accuracy for several gazes especially for downward and forward movements that caused by failure tracking mechanisms. Failure tracking mechanism mostly caused by failure pupil detection and tracking processes. According to the conducted research, there is a relation between eyeball movement and eyelid movement. Due to the relation, it can be concluded that the movement of the eyeball can be detected by utilize the eyelid movement without using the tracking mechanism. Hence, in this research, we propose an approach to detect the five movements of the eyeball using Eye-mark based on contour and edge detection. Our proposed method produces a brisk result with $91,2 \%$ of accuracy and better computational time compared with other marking methods. Accordingly, it is interesting on how to relate between eyeball movements and electric wheelchair navigation mechanism.
\end{abstract}

Keywords: Eyeball, Eyelid, Landmark, Eye, Wheelchair, Disability, Key-point, Movement.

\section{Introduction}

A wheelchair is equipment needed by people with disabilities for navigating from one place to another. By using a wheelchair, people with disabilities can move and navigate using their hand muscles by rotating the wheels called manual wheelchairs [1].

A manual wheelchair is still the best low-cost equipment used widely for people with disabilities. But, not all people with disabilities can utilize the manual wheelchair. A manual wheelchair problem arises when the disability user of a wheelchair is not capable for a move and operate their hands for navigating the wheelchair, especially for people with multiple disabilities that can not move their feet and their hands wholly [2]. Therefore, in recent years, many inventions were done to solve problems such as one hand drivable manual wheelchair that aimed for people with a stroke that can only move one of their arms [3]. However, one hand drivable manual wheelchair still not a solution for people with multiple disabilities that can not operate their feet and arms hands wholly.
Another invention is using the electric wheelchair that operated by using a motor to propel the seated user invented by George Klein [4]. Advanced studies and invention also invented as an advanced version of electric wheelchair such as Electric wheelchair that controlled using joystick [5]. However, the joystick controller that operates by a finger is still needed the hand muscle movements [6].

People with multiple disabilities need special attention, need to remember, they are not capable to operate their hands and feet. Thus, in this situation, any invention of wheelchair and electric-wheelchair types that controlled by hand can not be applied for multiple disabilities navigation equipment devices.

A solution also proposed by using Electromyograms, Electrooculargrams, and Electroenchepalograms that utilize several electrodes that stick to the skin user to control the electricwheelchair [7-9]. The idea of these proposed solutions is to read the amount of electricity produced by muscles especially when people with disabilities try to move their arms. However, sticking electrodes 


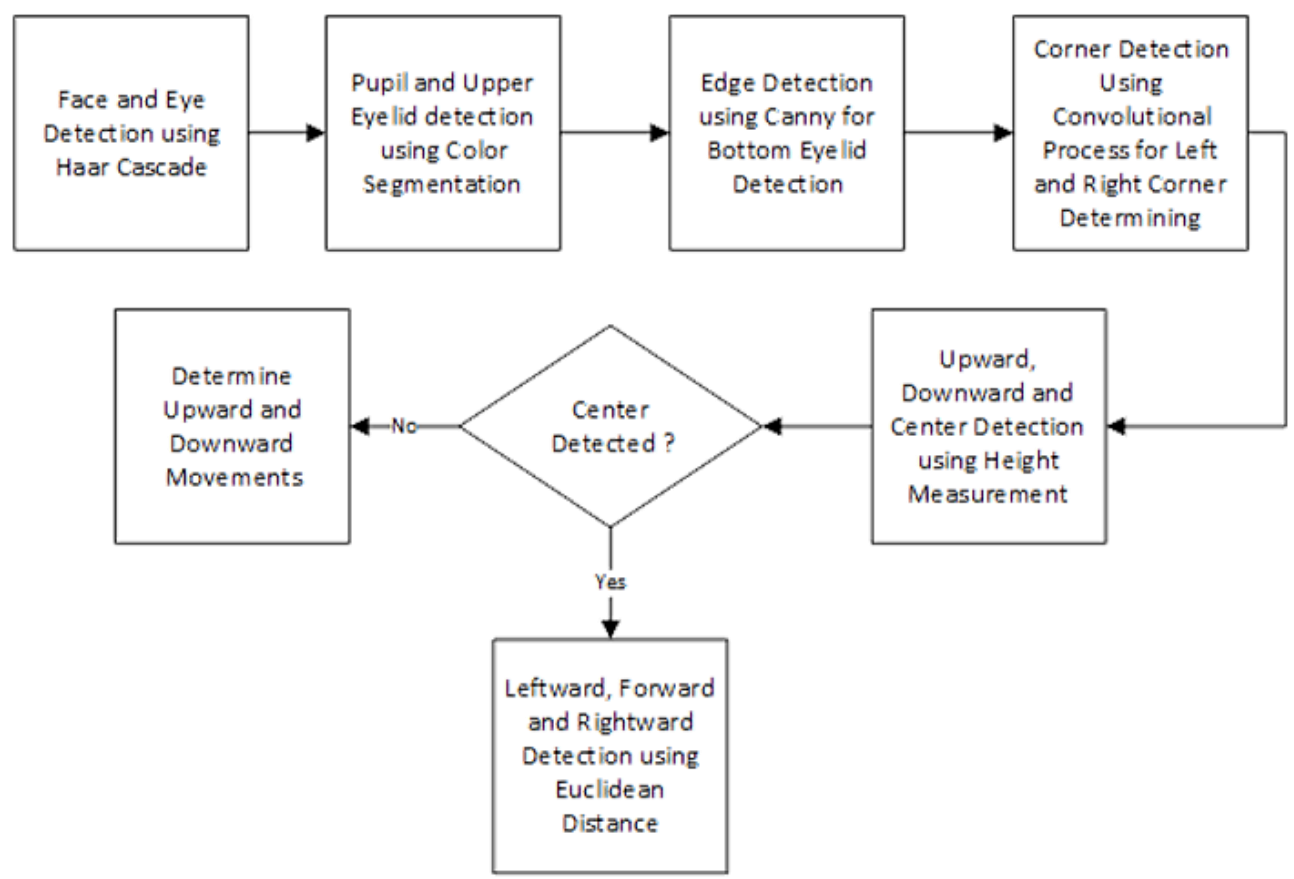

Figure. 1 Eyeball movements detection flow diagram

can be complicated because it needs assistance to stick it on appropriately into the skin, so the electrodes can read the electricity produced by arm muscles.

Another solution was proposed by using voice to be applied as an electric wheelchair controller [1012]. Nevertheless, a noisy environment can generate incorrect command considering that voice commands depending on the clarity pronounced by the user. Indeed, this problem can be solved by using a wearable microphone installed in the head, however, it still needs assistance for wearing it.

Therefore, we purpose an approach to detect and tracks the movements of the eyeball for navigating the wheelchair by using digital image processing that utilizes camera sensors. Camera sensors have an advantage especially for its ergonomics, by using a camera installed on the electric-wheelchair, it does not need wearing any wearable electrodes. By using camera and several digital image processing mechanisms, we also utilize the eyelid movements that has a relation to the eyeball movement to detects vertical movement such as upward, forward and downward. Also, horizontal movement such as rightward and leftward movement can be detected by utilizing distance between pupil and the edge side of the eyes.

In this article, the previous works of eyeball movement detection using camera sensors are discussed in the Section 2. In the Section 3, steps and methodologies to detects the movement of the eyeball is presented. Also in the Section 4, the result and

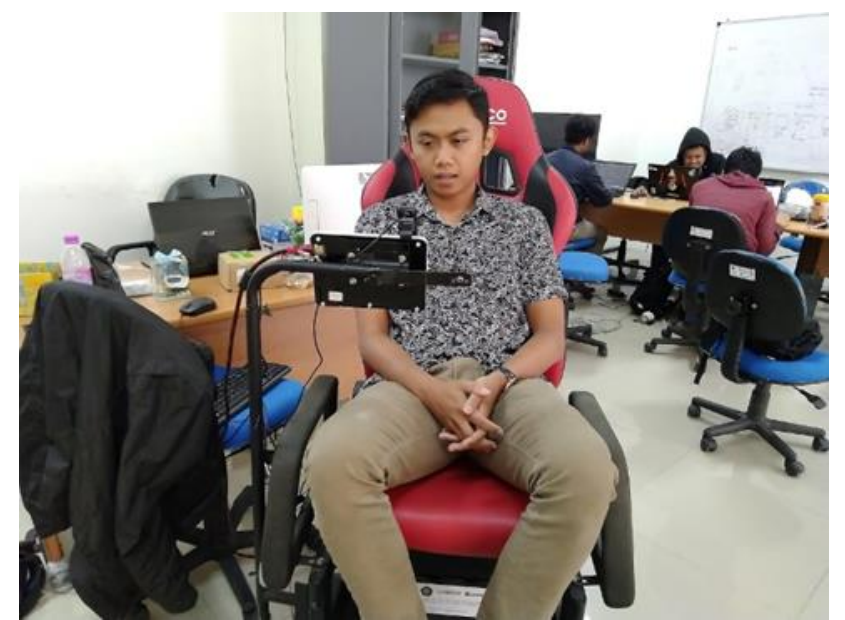

Figure. 2 Electric wheelchair ergonomics

discussion part is being included followed with conclusion described in Section 5.

\section{Previous work}

Plenty of research about eyeball movement detection has been done. The research proposed by Singh et al [13] uses eyeball movements as an electric wheelchair navigation controller. In his proposed research, sector measurement used to determine the directions of the eyeball movements by dividing the eye region into 3 sectors and compute the amount of the pixel in every region and then compared those 3 values to get the highest sector values. The highest values itself represent the directions of the eyeball movements. Nonetheless, this proposed method only can detect 3 directions of the eyeball movements that is leftward, rightward and forward. 
A method for eyeball movement detection also proposed by Patel et al [14]. In this research, circle detection was used to detect the pupil locations. Also, canny edge detection was used to detects the edge and size of the pupil itself. Nonetheless, this proposed method also can only detect 3 directions of the eyeball movements including forward, leftward and rightward.

Improvements result in eyeball movement detection also produced by Prasetya et al [15]. Using the Triangle Similarity method, 4 gaze directions of eyeball movements can be detected including upward, downward, leftward and rightward. Nonetheless, the downward detection still has lacks of accuracy. The lacks of downward accuracy is caused by the lack of ability for system to detects the vertical movement especially for downward movement detection. It is because, the angle produced by Triangle Similarity method for downward movement is quite similar with any other gaze. The lack of the accuracy of downward detection has been improved using Naive Bayes classification to determines the downward and upward movements [16]. The Naïve Bayes method is used to classified the vertical movement including upward and downward using angle values and Eye Aspect Ratio (EAR) as features. Therefore, downward movement can be distinguished with any other gazes due to the features produced.

However, 4 gazes of eyeball movement detection only produce four control mechanisms for wheelchair navigation control including upward detection for speed adjustment (speed up), downward detection for speed adjustment (slow down speed), rightward for turn right, and leftward for turn left. According to those navigation menus, forward detection was needed to determine the neutral position. The neutral position is required to avoid the confusing movements of the eyeball. As an example, when the user just looking forward to checking the terrain to be traversed, if there is no forward detection mechanism, a system will choose the most suitable possibilities whether upward, downward, leftward or rightward. Therefore, the decision taken by the system will influence the wheelchair movements.

Several research such as using Region Division [17] and Geometry Approach [18] also proposed 5 gaze eyeball movement detection. However, those methods still lack in the forward movement detection accuracies.

According to those problems, we proposed an eyeball movement mechanism to control the electric/smart wheelchair-using 5 directions of movements including upward, downward, forward, leftward and rightward.

\section{Methodology}

In this research, several processes of digital image processing and computational process are used to determine the direction movements of the eyeball as shown in Fig. 1.

\subsection{Face and eye location detection}

The first proses for detecting eyeball movements is to detect the face location. There are several ways to find the face location. The most commonly used method is using the Haar Cascade process proposed by Viola and Jones $[19,20]$. Also, there is another approach to detect the face location by using a Histogram of Gradient (HoG) [21, 22], until using the Convolution Neural Network (CNN) [23]. CNN produces an accurate detection for face location compared with Haar Cascade and HoG. However, CNN needs a multiple convolution process for Neural Network Classification schemas. Therefore, it will produce more computational processes rather than using Haar Cascade and $H o G$.

Due to our electric wheelchair user interaction mechanism, it is only driven by one user and the position of the user is relatively statics as shown in Fig. 2. Therefore, we do not use $\mathrm{CNN}$ as a face detection algorithm according to its slower computational times [24]. Therefore, Haar Cascade and $H o G$ are interesting choices for face and eye location detection.

Both Haar Cascade and $H o G$ produce a high frame rate rather than $\mathrm{CNN}$. Interestingly, Haar Cascade methods produce more accuracy compared with $H o G$ for object recognition [25]. Based on those results, we used Haar Cascade as the face and eye location detection.

In this research, we use images data that we have collected before. Each image is captured using a 1.3 MP camera resolution. Using Haar Cascade, the face area has been located by utilizing Haar features assisted with Integral Image to reduce computational

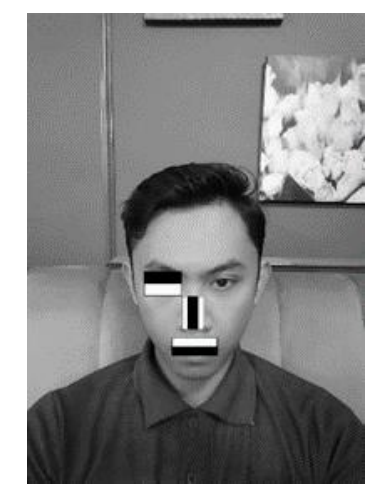

Figure. 3 Haar cascade illustration using Haar features 


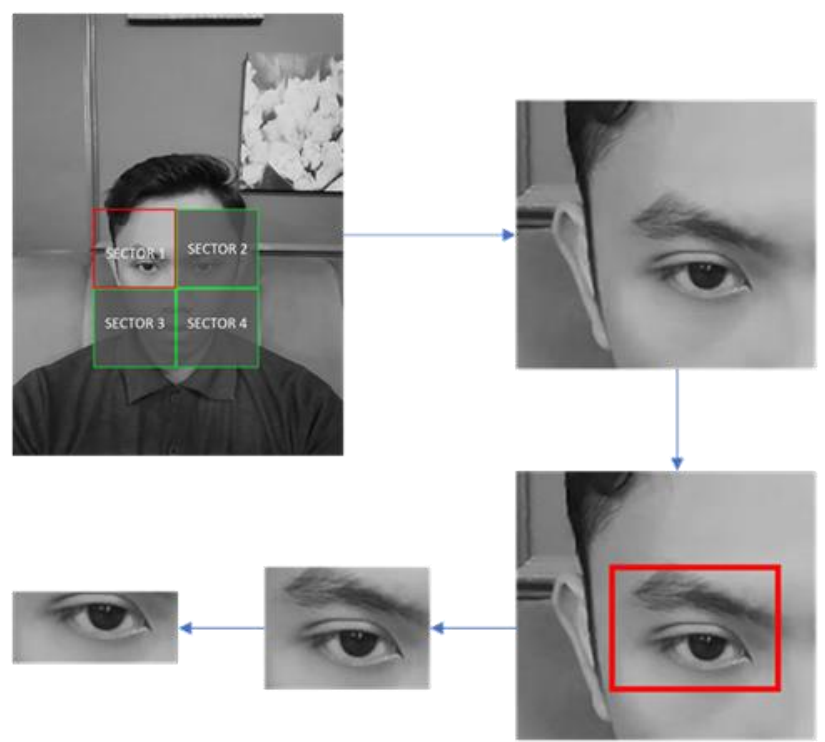

Figure. 4 Eye selection process

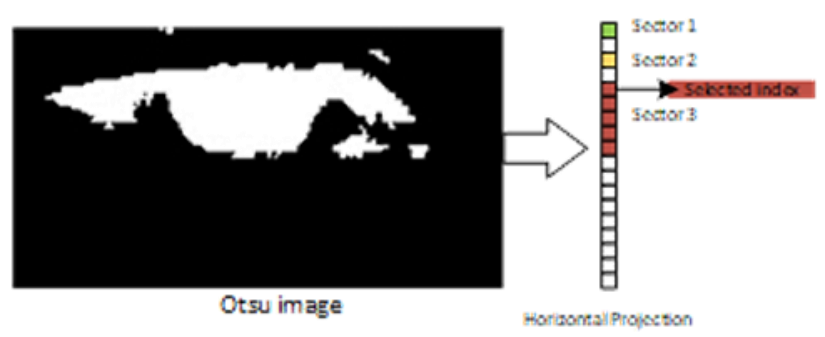

Figure. 5 Sectors generated using the Horizontal projection method

times as shown in Eq. (1):

$$
\int(x, y)=\sum_{x^{\prime} \leq x, y \prime \leq y} i\left(x^{\prime}, y^{\prime}\right)
$$

For instance, $\int(x, y)$ is the integral image, and $i\left(x^{\prime}, y^{\prime}\right)$ are the values of the original image at the location $(x, y)$.

Using the "cascading" process, an image window will be determined as a "face" area if the window can go through the whole cascading mechanism [26]. Haar Cascade process illustration was shown in Fig. 3.

Similar to the face location detection, the eye location detection process is using the same process as the face location detection did. The difference is the sliding window scanning process does not scan entirely image/frame, but scanning in the inside face location that detected on the previous detection. Also, we restrict the scanning area of the eyes location by dividing the face area into 4 sectors and do a scan in sector 1 only. After eye location is detected in sector 1 , we also crop the unnecessary eyebrows part so it will focus only on the eye region as shown in Fig. 4.

\subsection{Pupil and upper eyelid detection}

After eye location is detected, the next step is to detect the upper eyelid. According to the research by Plochl et al, eyelid movements are affected by pupil and eyeball movements [27]. Therefore, in this proposed method we assume that upper eyelids can be located by detecting the upper location of the pupil.

To detect the upper position of the pupil area, in this research we use a segmentation approach based on the Otsu threshold [28]. Therefore, the Gaussian filter was applied to the eye image using Eq. (2):

$$
G(x, y)=\frac{1}{2 \pi \sigma^{2}} e^{-\frac{e^{2}+y^{2}}{2 \sigma^{2}}}
$$

Denotes, $G(x, y)$ is the Gaussian threshold and $\sigma$ is the standard deviation. Afterward, the Otsu threshold was applied to the Gaussian image by using Eq. (3):

$$
\sigma_{w}^{2}(t)=w_{0}(t) \sigma_{0}^{2}(t)+w_{1}(t) \sigma_{1}^{2}(t)
$$

For instance, $\sigma_{w}^{2}(t)$ is the class variance, $w$ is the weight generated using Eq. (4).

$$
w=\sum_{i=0}^{\theta-1} p(i)
$$

Denotes, $\theta$ is the variable depends on the $w_{1}$ or $w_{2}$. For $w_{1}, \theta$ is $t$, and for $w_{2}, \theta$ is $L$ (histogram).

After the Otsu threshold process was done, the horizontal feature extraction process is applied to the Otsu threshold result image using Eq. (5):

$$
P(b)=\sum_{j=1}^{N} i\left(b, k_{j}\right)
$$

Denotes, $N$ is the weight of matrix/image, $b$ is the row, and $P$ is the horizontal projection result in the $k$ column. The result of horizontal projection will produce an array with length as the same as the height of the Otsu threshold result image. Following the Otsu threshold result, pupil area segmentation will generate a larger white color. However, the threshold result will not produce a clear result without noises as we expected. As shown in Fig. 5, horizontal projection results produce 3 different sectors. Each sector contains an array of columns that coincide and have values greater than 0 . Therefore, the highest sector must be selected, in this research, we use a computational work to select the highest sector and determine the upper side of the pupil by using the first 
index number of the highest sector value as a vertical coordinate of the upper eyelid.

\subsection{Bottom eyelid detection}

A bottom eyelid coordinate can be determined using the last index of the highest sector value that measured using Horizontal Projection before. Nevertheless, this approach cannot be applied if a pupil of the eyes does not intersect with the bottom eyelid, especially when eyeball looking upward. Therefore, for bottom eyelid detection, we use Canny Edge detection that represents in the Eq. (6).

$$
|G|=\sqrt{I_{x}^{2}+I_{y}^{2}}
$$

Denotes, $|G|$ is the gradient intensity, where $I_{x}$ and $I_{y}$ are the convolved image using $k_{x}$ and $k_{y}$ respectively (Eq. (7) and Eq. (8)).

$$
\begin{gathered}
k_{x}=\left[\begin{array}{ccc}
-1 & 0 & 1 \\
-2 & 0 & 2 \\
-1 & 0 & 1
\end{array}\right] \\
k_{y}=\left[\begin{array}{ccc}
1 & 2 & 1 \\
0 & 0 & 0 \\
-1 & -2 & -1
\end{array}\right]
\end{gathered}
$$
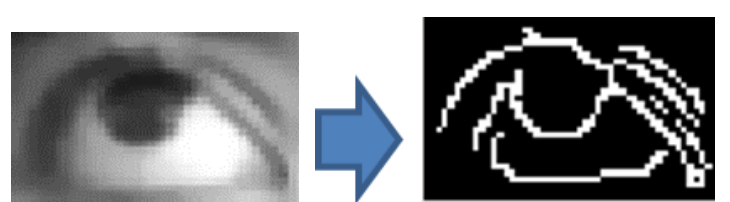

Figure. 6 Canny edge detection result from Gaussian blur image
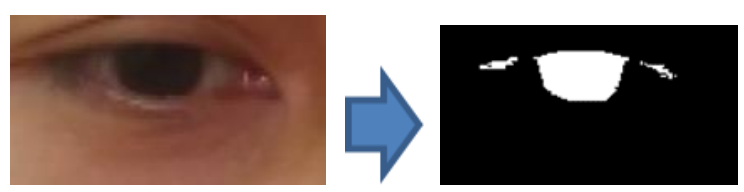

(a)
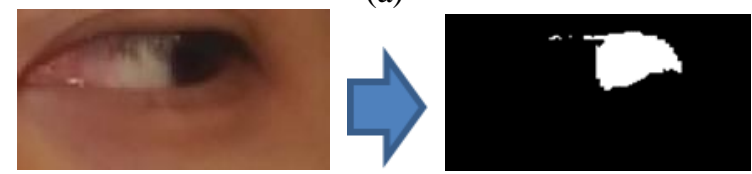

(b)

Figure. 7 Otsu threshold result does not represent the edge and corner of the eyes: (a) eyeball looking forward and (b) eyeball looking leftward

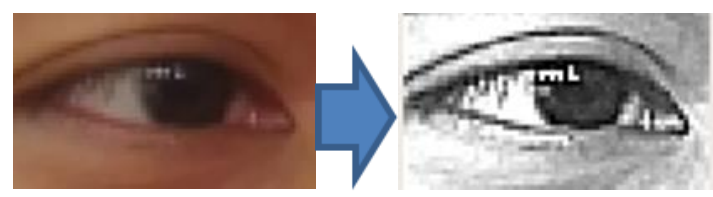

Figure. 8 Convolution result for edge sharpening
To minimize the resulting edge caused by skin scratches, Canny Edge detection was applied to Gaussian blur image generated before (Eq. (2)). The result of Canny Edge detection was shown in Fig. 6.

\subsection{Left and right corner detection}

Left and right corner detection are needed to determine the corner of the eyes. Using the left and right corner coordinate, the horizontal movements of the eyeball can be detected. Slightly different from upper eyelid detection, sector mechanism can not be applied due to the not optimal Otsu threshold for edge and corner detection result especially when the eyeball is not right in the middle as shown in Fig. 7.

Accordingly, the edge sharpening method is needed. The filtering approach using the convolution method is applied to the grayscale image of eye location by using a $5 \times 5$ matrix kernel as shown in Eq. (9).

$$
G(x, y)=(f * h)[x, y]=\sum_{j} \sum_{k} h[j, k] f[x-j, y-k]
$$

Denotes, $f$ is the input image, $x$ and $y$ is the index of rows and column respectively, and $h$ is the filter or kernel generated using Eq. (7).

$$
h=\left[\begin{array}{ccc}
-1 & -1 & -1 \\
-1 & 10 & -1 \\
-1 & -1 & -1
\end{array}\right]
$$

Convolution process will generate a binary image with sharped edges. The result of the convolution process is cropped with upper eyelid as an upper edge of the image as shown in Fig. 8.

After the convolution process, the next process is to select the right and left edge. In this research, we used a Vertical Projection method to sum the pixel based on the column. The Vertical Projection result will produce an array, using those array, the left and right edge of the eye location can be determined using the first and the last of a sector that contains values greater than 0 as shown in Fig. 9 .

\subsection{Upward, forward, and downward detection}

By utilizing the result of upper eyelid detection, bottom eyelid detection, and left and right corner detection, we can determine the boundary of the eye using those key-point coordinates, we also call it the Eye-mark approach. 


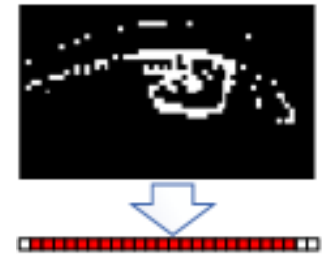

Figure. 9 Threshold result after convolution process

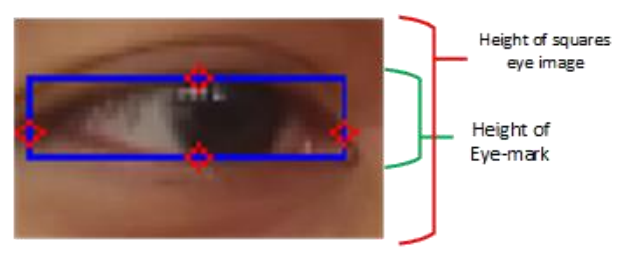

Figure. 10 The eye-mark result using Contour and Edge detection

In this research, we propose a method to detect upward, forward and downward movements of the eyeball by using the comparison between the height of Eye-mark and height of square eye image called Height Ratio, shown in Fig. 10. and explained in Eq. (11).

$$
E s=\left\{\begin{array}{c}
h>\sigma_{u}, \text { upward } \\
\mu_{d}<h<\sigma_{u}, \text { forward } \\
h<\mu_{d}, \text { downward }
\end{array}\right.
$$

Denotes, $\sigma_{u}$ is the smallest of height value when eyeball looking upward, $\mu_{d}$ is the highest of height value when eyeball looking downward, and $h$ is the height value of Eye-mark determined using Eq. (12).

$$
h=\frac{H_{e}}{H_{T}}
$$

For instance, $H_{e}$ is the height of the marked square (Eye-mark) and $H_{T}$ is the height of the eye selection process (Fig. 4).

\subsection{Leftward, forward, and rightward movements detection}

By using height measurement, it can be determined the vertical movements of the eyeball including upward, forward and downward movements. Therefore, it needs an extra process to determine the horizontal movements of the eyeball like leftward, forward, and rightward. In this research, we assume that horizontal movements of the eyeball can occur when the height value of the Eye-mark is "forward".
Leftward, forward and rightward detection mechanism is processed using the left and right horizontal coordinates that generated from the left and right corner detection process (Fig. 10). The left and right coordinates will be the point of reference as same as center coordinate that generated from the average calculation between left and right, as shown in Fig. 11.

Referring to Fig. 11, the eyeball horizontal movements can be measured using Eq. (13).

$$
D=\min \left(L_{1}, L_{2}, L_{3}\right)
$$

Denotes $D$ is the direction of eyeball horizontal movements (leftward, forward and rightward), $L_{1}$ is a distance between the center of the pupil to the right point, $L_{2}$ is a distance between the center of the pupil to the center point, and $L_{3}$ is a distance between center of pupil with left point coordinates.

The center of the pupil can be measured using the Otsu threshold process. The Otsu threshold process produces a binary image containing a shape, therefore, a center of the pupil will be referring to the larger shape in the Otsu threshold image. In this research, $a$ Centroid approach is applied to determine the center of the pupil using Eq. (14).

$$
(x, y)=\left[\left(x \rightarrow X_{\alpha}+R_{t}\right),\left(y \rightarrow Y_{\delta}+R_{\delta}\right)\right]
$$

For instance, $x$ and $y$ are the coordinates of the pupil, $X_{\alpha}$ is the beginning of weight, $Y_{\delta}$ is the beginning of

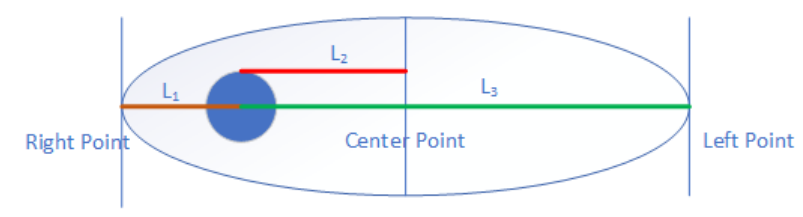

Figure. 11 Eyeball movement illustration using distance point method

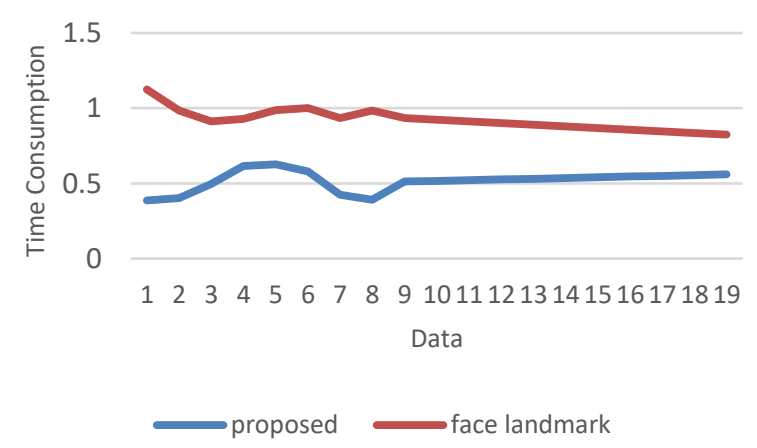

Figure. 12 Computational time comparison of the proposed method and face landmark method to determine the eye marking positions. 


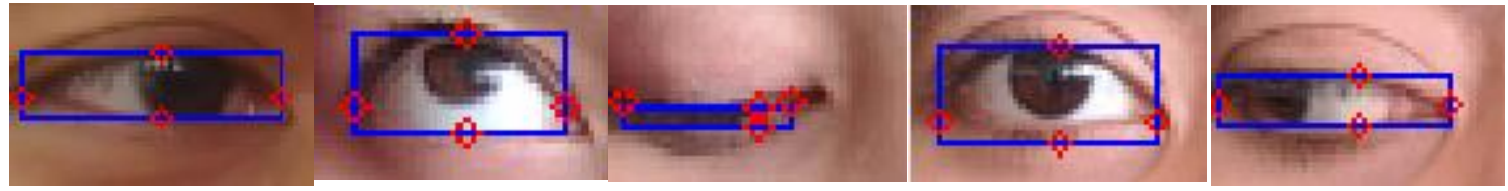

(a)
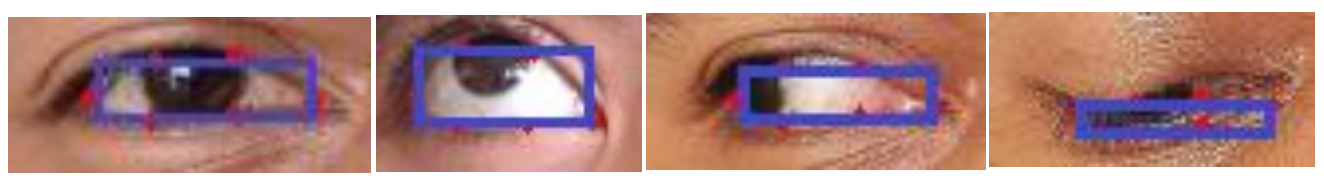

(b)

Figure. 13 Key-point generates a result: (a) using the proposed method and (b) using face landmark method

height, while $R_{t}$ and $R_{\delta}$ were generated using Eqs. (15) and (16).

$$
\begin{array}{r}
R_{t}=\frac{\sqrt{\left(x_{\alpha}-x_{\beta}\right)^{2}+\left(y_{\alpha}-y_{\beta}\right)^{2}}}{2} \\
R_{\delta}=\frac{\sqrt{\left(x_{r}-x_{\delta}\right)^{2}+\left(y_{r}-y_{\delta}\right)^{2}}}{2}
\end{array}
$$

Denotes, $x_{\alpha}$ and $y_{\alpha}$ are the beginning shape coordinate of the vertical direction, $x_{\beta}$ and $y_{\beta}$ are the ending shape coordinate of the vertical line. $x_{\delta}$ and $y_{\delta}$ are the beginning shape coordinate of the horizontal direction and $x_{r}$ and $y_{r}$ are the ending shapes of horizontal directions.

\section{Result and analysis}

We proposed an approach to detects the eyeball movement directions using Eye-mark based on edge and contour. The output of this research is the marking point in the eye area especially on the upper, bottom, left and right corner for detects the movements of the eyeball.

In this research, we use 50 different person face data that captured manually using 1.3 MP of camera resolution for trial process. Every person or respondence asked for moves their eyeball with accordance of 5 gaze movement. The total image data that we are used, is 250 images data including 50 images for every movement.

Another research also uses a marking point to detects the blinks movement of the eyes called Facial Landmark [29]. Facial Landmark is the method of marking the face area using the Tree Cascade method proposed by Kazemi et al [30]. The problem of the Facial Landmark approach is the use of high computational time. In this research, we also compare the computational time needed between our proposed method and the Facial Landmark method to detects the eye locations using 20 images data as shown in Fig. 12.

According to Fig. 12, our proposed method can overcome the Face Landmark methods for computational times. It is because our proposed method does not use a gradual method like Tree Cascade. Our proposed method only using image processing to determine the eye marking position. Several results of Eye-mark as proposed method and facial landmark method for determining the eye marking coordinates also shown in Fig. 13.

About 150 data of horizontal eye movements are tested to gain the Height Ratio including 50 data for downward, 50 data for forward and 50 data for upward. The result of the Height Ratio measurement is shown in Fig. 14.

Referring to Fig. 14, there is a difference evident between upward, forward and downward state. As mentioned in Eq. (11), the highest value of downward $\left(\mu_{d}\right)$ is represented with a blue ocean straight line, and the smallest value of upward $\left(\sigma_{d}\right)$ is represented with a green light straight line.

Table 1. Detection result using the proposed method

\begin{tabular}{|c|c|c|c|}
\hline Directions & Correct & False & $\begin{array}{c}\text { Accuracy } \\
(\%)\end{array}$ \\
\hline Upward & 45 & 5 & $90 \%$ \\
\hline Rightward & 46 & 4 & $92 \%$ \\
\hline Forward & 46 & 4 & $92 \%$ \\
\hline Leftward & 44 & 6 & $88 \%$ \\
\hline Downward & 47 & 3 & $94 \%$ \\
\hline \multicolumn{4}{|c|}{ Average } \\
\end{tabular}




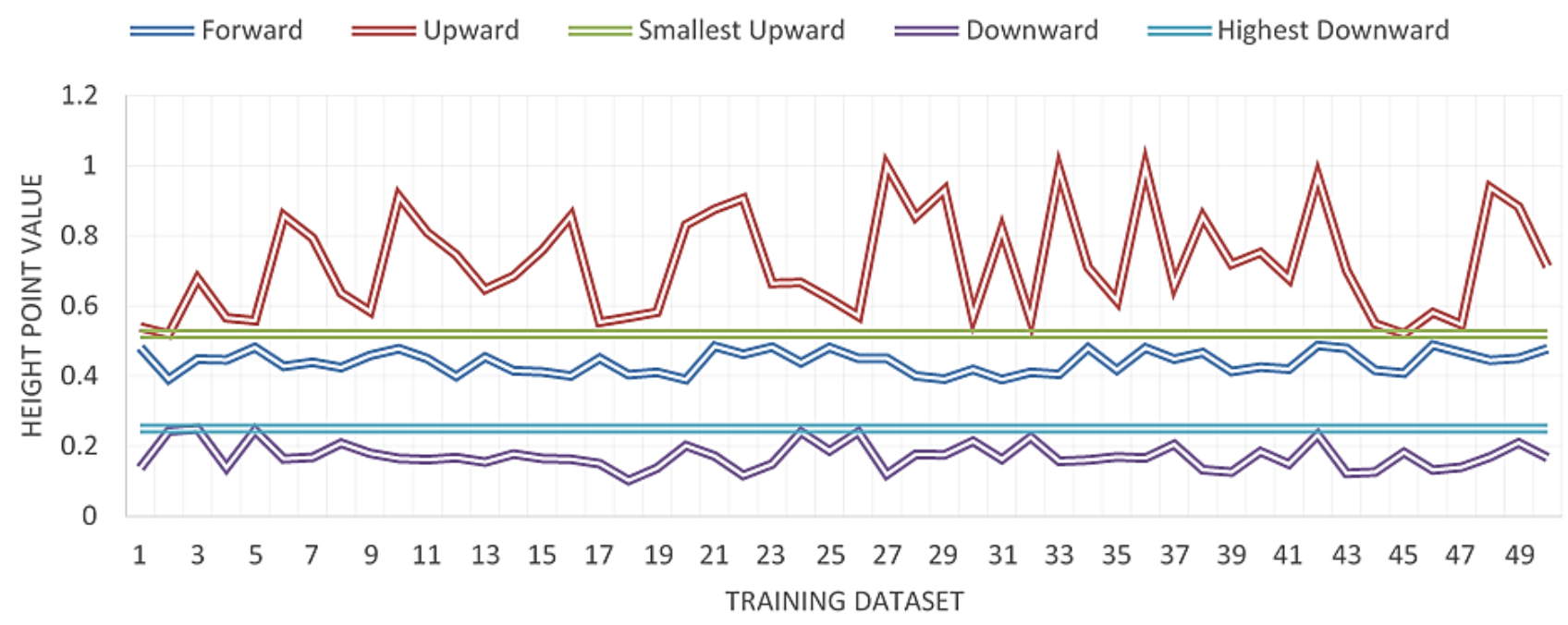

Figure. 14 Height Ratio measurement result for training dataset

Table 2. Comparison of our proposed method with Triangle Similarity and Naïve Bayes Triangle Similarity methods

\begin{tabular}{|c|c|c|c|}
\hline \multirow{2}{*}{ Directions } & \multicolumn{3}{|c|}{ Detection Accuracy (\%) } \\
\cline { 2 - 4 } & TS [15] & $\begin{array}{c}\text { NBTS } \\
{[16]}\end{array}$ & Proposed \\
\hline Upward & $75 \%$ & $80 \%$ & $\mathbf{9 0} \%$ \\
\hline Downward & $58,3 \%$ & $80 \%$ & $\mathbf{9 4} \%$ \\
\hline Rightward & $91,6 \%$ & $90 \%$ & $\mathbf{9 2} \%$ \\
\hline Leftward & $91,6 \%$ & $90 \%$ & $\mathbf{8 8} \%$ \\
\hline Average & $79,12 \%$ & $85 \%$ & $\mathbf{9 1 \%}$ \\
\hline
\end{tabular}

Our proposed method also tested to detects the movements of the eyeball to 50 respondents. Every respondent also asks to move their eyeball to the five directions includes upward, forward, downward, leftward and rightward. Total data for testing is 250 data captured using the same hardware as train data. The result of the experiment is shown in Table. 1.

Our proposed method also compared with another previous method like Region Division (RD) method [17], Triangle Similarity (TS) [15], Nä̈ve Bayes Triangle Similarity (NBTS) [16], and Coordinate Geometry (CG) method [18].

In the first comparison section, our proposed method was compared with Triangle Similarity and Nä̈ve Bayes Triangle Similarity methods with focusing on upward, downward, leftward and rightward movements detection shown in Table 2.

According to Table 2, our proposed method can outperform the two other methods. Our proposed method can detect upward, downward, leftward and rightward movements around $91 \%$ of accuracy. TS and NBTS method has lower average detection accuracy caused by circle detection problem. TS and NBTS method experience difficulty for pupil detection using circle detection mechanism, it is because a pupil does not visible as a prefect circle when several parts of pupil is covered by eyelid, especially for vertical movement such as upward and downward.

Our proposed method also compared with the Coordinate Geometry method and Region Division method to shows the ability for forward movement detection as shown in Table 3 .

Referring to Table 3, the results of forward eyeball movements detection generated by Region Division and Coordinate Geometry approach is surpassed by our proposed method. Our proposed method is generating $92 \%$ of forwarding movement detection using the proposed Distance Point method (Fig. 11). There are several reasons of why our proposed method is superior, in the Region Division method, there is a registration mechanism before the user can use the eyeball detection system. This mechanism only saves the registration data in the current state. Therefore, if there is some movement of the body such as come forward or away from the camera, the existing conditions or state will be different with the saved state before, especially for forward movement as the center of the eyeball movement direction. Slightly different from Region Division method, Coordinate Geometry approach has a high sensitivity issues caused by their mechanism that compare the movement with the initial state. Therefore, if there is a little movement of the eyeball, it will be detected as the movement without providing the appropriate range of the movement. 
In the last, our proposed method is compared with the Region Division method to shows the performance of 5 gaze eyeball movements as shown in Table 4. Our proposed method has a bit of excellence compared with the Region Division method. Our proposed method has $91,2 \%$ accuracy greater than $91 \%$ of accuracy generated by the Region Division method. Furthermore, our proposed method does not require any calibration process required by the Region Division method. Several samples of correct detection results generated by our proposed method also shown in Fig. 15. As an approach, our proposed method also has lacks and deficiency, several results of failure detections result also shown in Fig. 16.

\section{Conclusion}

This research proposes an approach to detects five movements of the eyeball including upward, downward, leftward, forward and rightward. Our proposed method is utilizing the marker of the eye location called Eye-mark. An eye-mark method is the marking eye area approach by utilizing pure digital image processing approaches without involving any

Table 3. Comparison of our proposed method with Region Division and Coordinate Geometry methods

\begin{tabular}{|c|c|c|c|}
\hline Directions & \multicolumn{3}{|c|}{ Detection Accuracy (\%) } \\
\hline & RD [17] & $\begin{array}{c}\text { CG } \\
{[18]}\end{array}$ & Proposed \\
\hline Forward & $80 \%$ & $20 \%$ & $\mathbf{9 2} \%$ \\
\hline
\end{tabular}

Table 4. Comparison of our proposed method with the Region Division method to detects 5 directions of eyeball movements

\begin{tabular}{|c|c|c|}
\hline \multirow{2}{*}{ Directions } & \multicolumn{2}{|c|}{ Detection Accuracy (\%) } \\
\cline { 2 - 3 } & RD [29] & Proposed \\
\hline Upward & $85 \%$ & $90 \%$ \\
\hline Downward & $96 \%$ & $94 \%$ \\
\hline Forward & $80 \%$ & $92 \%$ \\
\hline Leftward & $97 \%$ & $88 \%$ \\
\hline Rightward & $97 \%$ & $92 \%$ \\
\hline Average & $91 \%$ & $\mathbf{9 1 , 2} \%$ \\
\hline
\end{tabular}

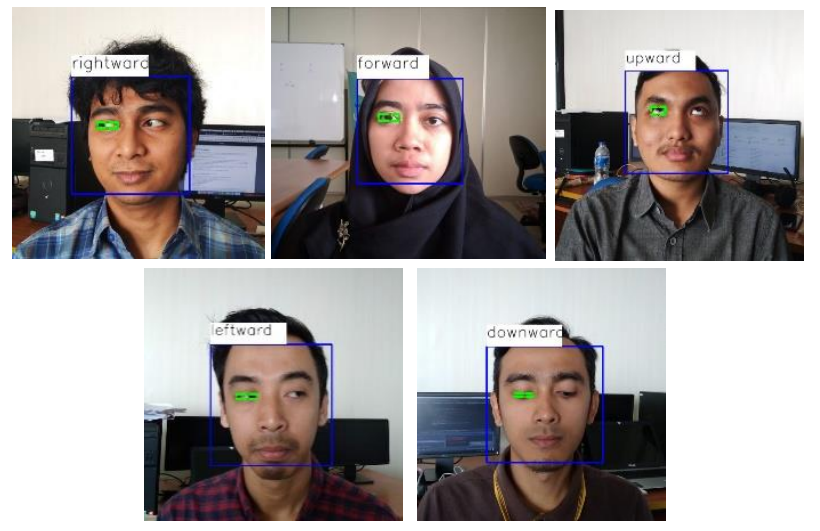

Figure. 15 Several correct detection results using our proposed method
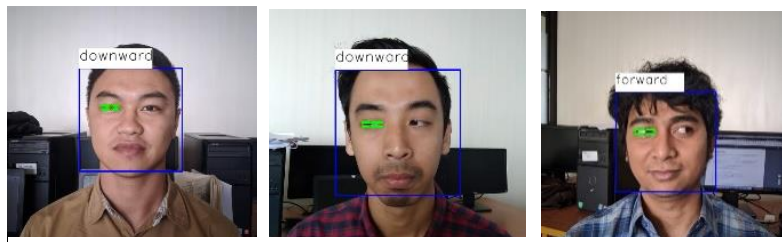

Figure. 16 Several samples of failure detection result

classification. Therefore, our proposed marking method can outperform the computation times generated by other marking methods and produce less time computation results. The smaller the computation time, the higher the FPS (Frame Per Second), the more information we can get.

Our proposed method also generates 91,2\% accuracy for eyeball movement detection. This proposed method can outperform any other methods and can perform well without any calibration process before. Therefore, our proposed method is a worthy approach for applied as an electric wheelchair control movements like upward for increasing speed, downward for decreasing speed, leftward and rightward for left and right movements and forward for straight forward movement.

\section{Acknowledgments}

This research is supported by Computer Vision Research Group, Faculty of Computer Science, Brawijaya University. Also, the author is very thankful for all respondents for allowing us to make use of their image in this research.

\section{References}

[1] V. Der Woude and L. Vincentius, "Manual Wheelchair Propulsion: An Ergonomic Perspective: Academisch Proefschrift...," Free University Press, 1989. 
[2] A. C. Bebech, T. D. Oliver, A. M. Limperos, B. P. Schade, and K. H. Larwin, "Perception of Students With Multiple Disabilities and Typically Developing Peers", European Journal of Spesial Education Reseacrh, Vol. 1, No. 3, pp. 1-11, 2016.

[3] H. S. Jung, G. Park, Y. S. Kim, and H. S. Jung, "Development and evaluation of one-hand drivable manual wheelchair device for hemiplegic patients", Applied Ergonomics, Vol. 48, pp. 11-21, 2015.

[4] R. Bourgeois-Doyle, George Klein, The Great Inventor. Ottawa: National Research Council of Canada Press, 2004.

[5] T. M. Nirmal, "Wheelchair for Physically and Mentally Disabled Persons", International Journal of Electrical and Electronics Research ISSN, Vol. 2, No. 2, pp. 112-118, 2014.

[6] R. H. Rozendal, "Human poly-articular muscles: An anatomical comment", Human Movement Science, Vol. 13, No. 5, pp. 557-568, 1994.

[7] R. Hardiansyah, "The Electric Wheelchair Control Using Electromyography Sensor Of Arm Muscle", International Conf. on Information, Communication Technology and System, pp. 129-134, 2016.

[8] C. Ishii, S. Murooka, and M. Tajima, "Navigation of an Electric Wheelchair Using Electromyograms, Electrooculograms, and Electroencephalograms", International Journal of Mechanical Engineering and Robotics Research, Vol. 7, No. 2, pp. 143-149, 2018.

[9] C. Atmaji, A. E. Putra, and A. Hanif, "Sliding window method for eye movement detection based on electrooculogram signal", In: Proc. of 2018 International Conf. on Information and Communications Technology, Vol. 2018-Janua, pp. 628-632, 2018.

[10] S. R. Avutu, "Voice control module for Low cost Local-Map navigation based Intillegent wheelchair", Vol. 2016 IEEE, pp. 1-5, 2017.

[11] N. Peixoto, H. G. Nik, and H. Charkhkar, "Voice controlled wheelchairs: Fine control by humming", Computer Methods and Programs in Biomedicine, Vol. 112, No. 1, pp. 156-165, 2013.

[12] D. Wang and H. Yu, "Development of the control system of a voice-operated wheelchair with multi-posture characteristics", In: Proc. of 2017 2nd Asia-Pacific Conf. on Intelligent Robot Systems, pp. 151-155, 2017.

[13] M. Singh, P. Jain, and S. Chopra, "Eye movement detection for wheelchair control application", In: Proc. of International Conf. on
Electrical, Electronics, Signals, Communication and Optimization, pp. 4-8, 2015.

[14] S. N. Patel and V. Prakash, "Autonomous camera based eye controlled wheelchair system using raspberry-pi", In: Proc. of 2015 IEEE International Conf. on Innovations in Information, Embedded and Communication Systems, pp. 3-8, 2015.

[15] R. P. Prasetya and F. Utaminingrum, "Triangle similarity approach for detecting eyeball movement", In: Proc. of the 5th International Symposium on Computational and Business Intelligence, pp. 37-40, 2017.

[16] G. Pangestu, F. Utaminingrum, and F. A. Bachtiar, "Eyeball Movement Detection System using Corner Triangle Similarity, Naïve Bayes , and Ear Approach", International Journal of Advances in Soft Computing and its Applications, Vol. 11, No. 2, pp. 1-14, 2019.

[17] R. P. Prasetya, F. Utaminingrum, and W. F. Mahmudy, "Real time eyeball movement detection based on region division and midpoint position", International Journal of Intelligent Engineering and Systems, Vol. 11, No. 3, pp. 149-158, 2018.

[18] M. Gneo, M. Schmid, S. Conforto, and T. D'Alessio, "A free geometry modelindependent neural eye-gaze tracking system", Journal of NeuroEngineering and Rehabilitation, Vol. 9, No. 1, 2012.

[19] P. Viola and M. J. Jones, "Robust real-time face detection", International Journal of Computer Vision, Vol. 57, No. 2, pp. 137-154, 2004.

[20] Mg. Krishna and A. Srinivasulu, "Face Detection System on AdaBoost Algorithm Using Haar Classifiers", International Journal of Modern Engineering Research, Vol. 2, No. 5, pp. 3556-3560, 2012.

[21] N. Rekha and M. Z. Kurian, "2014 Face Detection in Real Time Based on HOG_IJARCET-VOL-3-ISSUE-4-1345-1352", International Journal of Advanced Research in Computer Engineering \& Technology, Vol. 3, No. 4, pp. 1345-1352, 2014.

[22] H. S. Dadi and G. K. Mohan Pillutla, "Improved Face Recognition Rate Using HOG Features and SVM Classifier", IOSR Journal of Electronics and Communication Engineering, Vol. 11, No. 04, pp. 34-44, 2016.

[23] L. L. S. Deffo, E. Tagne Fute, and E. Tonye, "CNNSFR: A convolutional neural network system for face detection and recognition", International Journal of Advanced Computer Science and Applications, Vol. 9, No. 12, pp. 240-244, 2018. 
[24] V. Gupta, "Face Detection - OpenCV, Dlib and Deep Learning ( C++ / Python )", 2018. [Online]. Available: https://www.learnopencv.com/facedetection-opencv-dlib-and-deep-learning-cpython/.

[25] J. Cruz, E. Shiguemori, and L. Guimarães, “A comparison of Haar-like, LBP and HOG approaches to concrete and asphalt runway detection in high resolution imagery", Journal of Computational Interdisciplinary Sciences, Vol. 6, No. 3, pp. 121-136, 2016.

[26] M. S. Uddin and A. Y. Akhi, "Horse Detection Using Haar Like Features", International Journal of Computer Theory and Engineering, Vol. 8, No. 5, pp. 415-418, 2016.

[27] M. Plöchl, J. P. Ossandón, and P. König, "Combining EEG and eye tracking: Identification, characterization, and correction of eye movement artifacts in electroencephalographic data", Frontiers in Human Neuroscience, Vol. 6, No. OCTOBER 2012, pp. 1-23, 2012.

[28] R. Abiyev and K. Kilic, "Adaptive iris segmentation", Lecture Notes in Computer Science (including subseries Lecture Notes in Artificial Intelligence and Lecture Notes in Bioinformatics), Vol. 5576 LNCS, pp. 90-99, 2009.

[29] T. Soukupová, "Real-Time Eye Blink Detection using Facial Landmarks", In: Proc. of Computer Vision Winter Workshop, 2016.

[30] V. Kazemi and J. Sullivan, "One Millisecond Face Alignment with an Assemble of Regression Trees", In: Proc. of Conf. on Computer Vision and Pattern Recognition, 2017. 